NASA Technical Memorandum 102377

\title{
Effect of Spatial Resolution on Apparent Sensitivity to Initial Conditions of a Decaying Flow as it Becomes Turbulent
}

Robert G. Deissler and Frank B. Molls

Lewis Research Center

Cleveland, Ohio

November 1989

\section{NASN}


EFFECT OF SPATIAL RESOLUTION ON APPARENT SENSITIVITY TO INITIAL CONDITIONS OF A DECAYING FLOW AS IT BECOMES TURBULENT

Robert G. Deissler and Frank B. Molls National Aeronautics and Space Administration Lewis Research Center Cleveland, Ohio 44135

\section{ABSTRACT}

Grids with $32^{3}, 64^{3}$, and $128^{3}$ points are used in numerical. solutions for a decaying flow. The sensitivity of initially neighboring solutions to small changes in initial conditions increases as the spatial resolution improves. A fourth-order finite-difference method is used for the solutions with $32^{3}$ and $64^{3}$ grid points, and a pseudospectral method for $128^{3}$ grid points. The latter solutions appear to be rather wellresolved, in spite of the formation of steep velocity gradients in the flow.
47.20.Tg,
47.25. Ae,
$47.25 . \mathrm{Cg}$
$47.20 . \mathrm{Ky}$ 
In Ref. 1 we obtained numerical solutions for decaying Navier-Stokes flows. Although the higher Reynolds-number solutions indicated sensitive dependence on initial conditions, there may be some question about the effect of numerical resolution. In particular, some studies have shown that sensitivity to small changes in initial conditions (chaoticity) may decrease with improved spatial resolution, if the solutions are under-resolved. 2

Here we explore the effect of improved numerical resolution and numerical method on the chaoticity of solutions for decaying Navier-Stokes flows--that is, for flows described by the Navier-Stokes equations without energy input. The Navier-Stokes equations for an incompressible flow can be written as

$$
\frac{\partial u_{i}}{\partial t}=-\frac{\partial\left(u_{i} u_{k}\right)}{\partial x_{k}}-\frac{1}{\rho} \frac{\partial p}{\partial x_{i}}+v \frac{\partial^{2} u_{i}}{\partial x_{k} \partial x_{k}},
$$

where the pressure is given by the Poisson equation

$$
\frac{1}{\rho} \frac{\partial^{2} p}{\partial x_{\ell} \partial x_{\ell}}=-\frac{\partial^{2}\left(u_{\ell} u_{k}\right)}{\partial x_{\ell} \partial x_{k}} \text {. }
$$

The subscripts can have the values 1,2 , or 3 , and a repeated subscript in a term indicates a summation, with the subscript successively taking on the values 1,2 , and 3 . The quantity $u_{i}$ is an instantaneous velocity component, $x_{j}$ is a space coordinate, $t$ is the time, $\rho$ is the density, $v$ is the kinematic viscosity, and $p$ is the instantaneous pressure. As in Ref. 1 the initial velocity is given by

$$
u_{i}=a_{j} \cos q \cdot x+b_{i} \cos r \cdot x+c_{j} \cos s \cdot x,
$$

where

$$
\begin{gathered}
a_{i}=k(2,1,1), \quad b_{i}=k(1,2,1), \quad c_{i}=k(1,1,2), \\
q_{i}=(-1,1,1) / x_{0}, \quad r_{i}=(1,-1,1) / x_{0}, \quad s_{i}=(1,1,-1) / x_{0},
\end{gathered}
$$

$k$ is a quantity that fixes the initial Reynolds number at $t=0$, and $x_{0}$ is one over the magnitude of an initial wavenumber component. The initial 
pressure is not specified since it is calculated from Eq. (2). Equations (3) and (4) satisfy continuity, and Eqs. (1) and (2) insure that continuity is maintained. The boundary conditions are periodic, with a period of $2 \pi x_{0}$.

Cubical computational grids are used for the numerical calculations. For the $32^{3}$ and $64^{3}$ grid-point cases fourth-order spatial differencing and third-order Adams-Moulton predictor-corrector time differencing are used, as in Ref. 1. For $128^{3}$ grid points a pseudospectral method is used for calculating the spatial derivatives. Since a pseudospectral method is of high (approaching infinite) order, the $128^{3}$ grid-point case should give even better resolution than if it had used the lower fourth-order method. A second-order AdamsBashforth method is used for the time differencing in the pseudospectral code. As in Ref. 1, numerical stability limitations force the timewise resolution to be good. The emphasis here is therefore on the effect of spatial resolution. In Ref. 3 we showed, by calculating largest Liapunov exponents, that long-term asymptotic solutions for Navier-Stokes turbulence have sensitive dependence on initial conditions. As usually defined, the Liapunov exponents cannot be calculated for a decaying flow. Since it is not clear how Liapunov exponents might be meaningfully defined for decaying flows, we investigate the chaoticity of the latter, as in Ref. 1 , by simply perturbing the initial conditions a small amount and comparing the perturbed solutions with the unperturbed ones. Here we perturb the $a_{j}, b_{j}$ and $c_{i}$ in Eq. (4) by 0.1 percent, while maintaining continuity. For example the $a_{j}$ are perturbed from $k(2,1,1)$ to $k(2.002,1.001,1.001)$. The constant $k$ is taken to be 640.

Figure 1 shows the effect of spatial resolution on the indicated sensitivity of the flow to small changes in initial conditions. It is clear that improved resolution increases the sensitivity of the solution to small initial-condition changes; the perturbed solution breaks away from the 
unperturbed one sooner for the more highly resolved cases. Thus our solutions are in a range where the resolution is higher than that where increasing resolution decreases the sensitive dependence on initial conditions. This is a comforting observation since, if the result were otherwise, the observed chaos in our solutions might be due to inadequate numerical resolution.

The results in Fig. 2 indicate that the case with $128^{3}$ grid points is rather well-resolved spatially. This is particularly so because of the highorder accuracy of the pseudospectral method used. (The grid points are shown by symbols.) The resolution occurs in spite of some steep gradients in the flow. The tendency to form steep gradients at some points in the flow is, of course, a well-known property of turbulent flows, and evidently occurs as a steepening effect of the nonlinear terms in the Navier-Stokes equations.

\section{ACKNOWLEDGMENTS}

We would like to thank R. Mulac for calculating the $64^{3}$ grid-point case, and P. McMurtry, R. Metcalf, and J. Riley for furnishing the pseudospectral code used herein. 


\section{REFERENCES}

IR.G. Deissler, Rev. Mod. Phys. 56, 223 (1984).

2J.H. Curry, J.R. Herring, J. Loncaric, and S.A. Orszag, J. Fluid Mech. 147, 1 (1984).

3R.G. Deissler, Phys. Fluids 29, 1453 (1986).

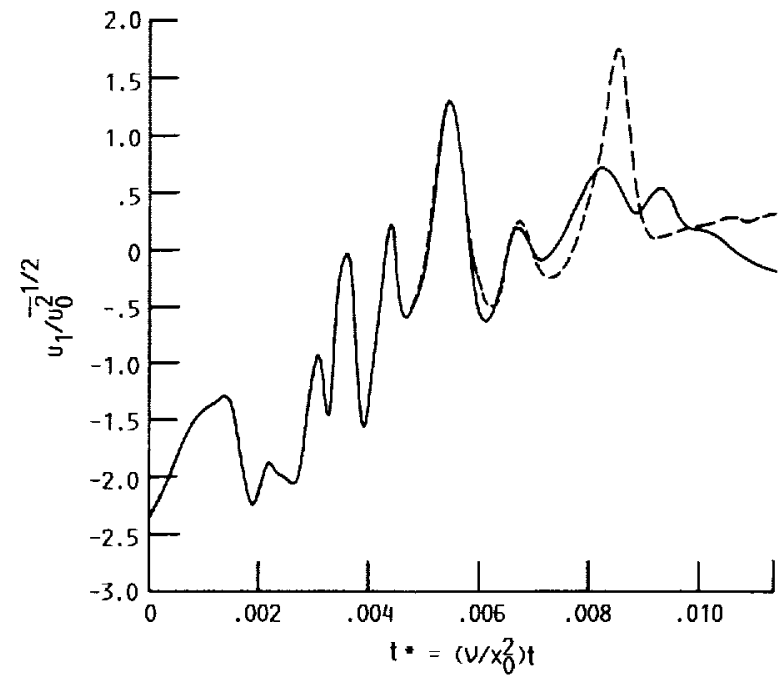

(a) $32^{3}$ GRID POINTS.

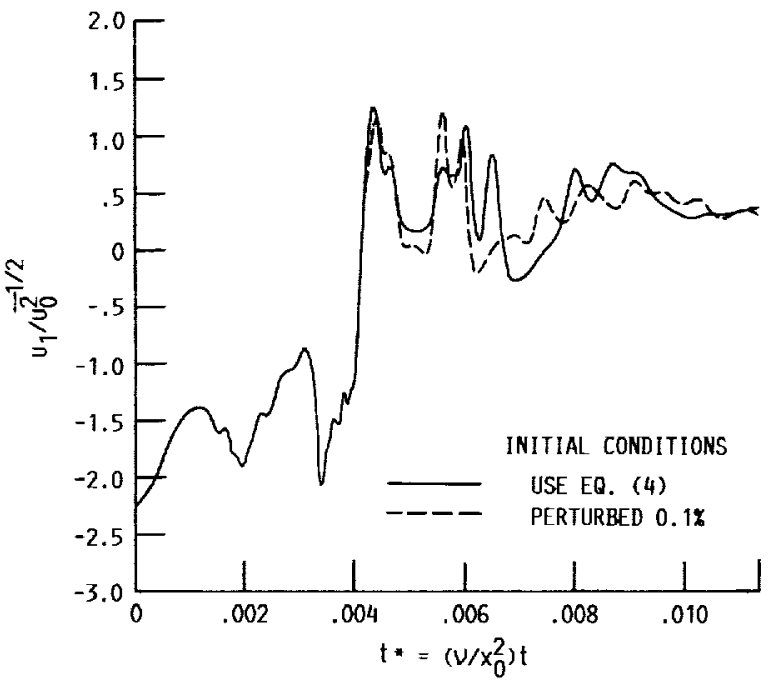

(b) $64^{3}$ GRID POINTS.

FIGURE 1. - CACILLATED EVOLUTION OF VELOCITY FLUCTUSTIONS AT GRID CENIER (NORMALIZED BY INITIAL CONDITIONS) FOR INITIAL REYNOLDS NUMBER

$\left(u_{0}^{2}\right)^{1 / 2} x_{0} v=1180(k$ IN EQ. (4) $=640)$. ROOT-

MEAN-SQUARE FLUCTUATIONS ARE SPATIALLY AVERAGED. 


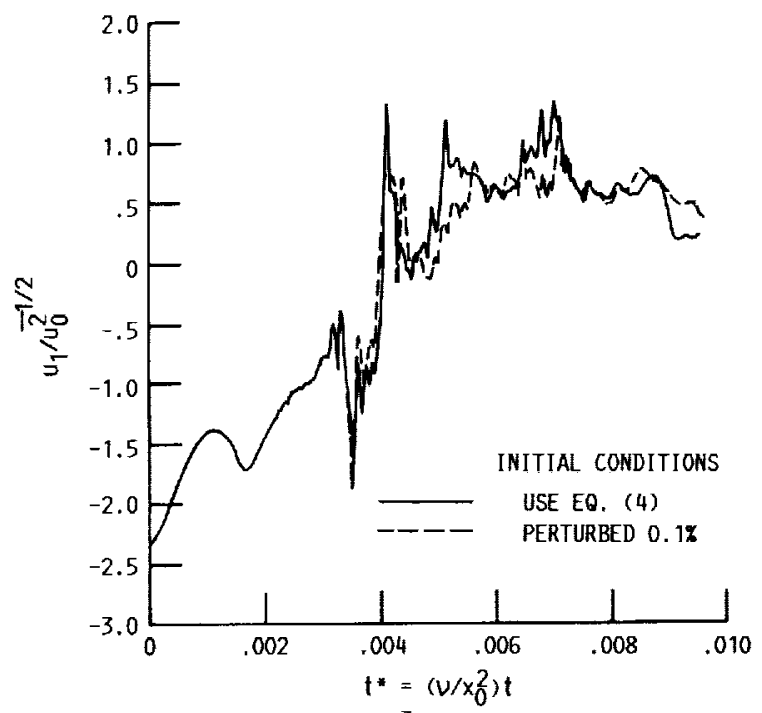

(c) $128^{3}$ GRID POINTS

FIGURE 1. - CONCLUDED.

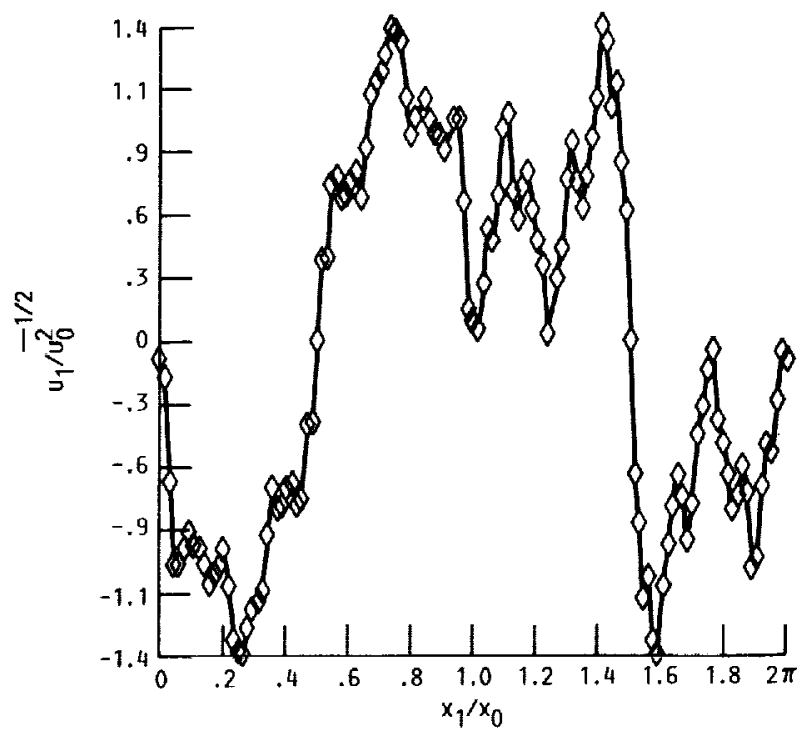

FIGURE 2 - CALCULATED SPATIAL VARIATION OF VELOCITY FLUCTUATIONS ON A PLANE THROUGH GRID CENTER, SYMBOLS ARE AT GRID POINTS. NUMBER OF GRID POINTS, $128^{3} . t^{*}=.0050$. 


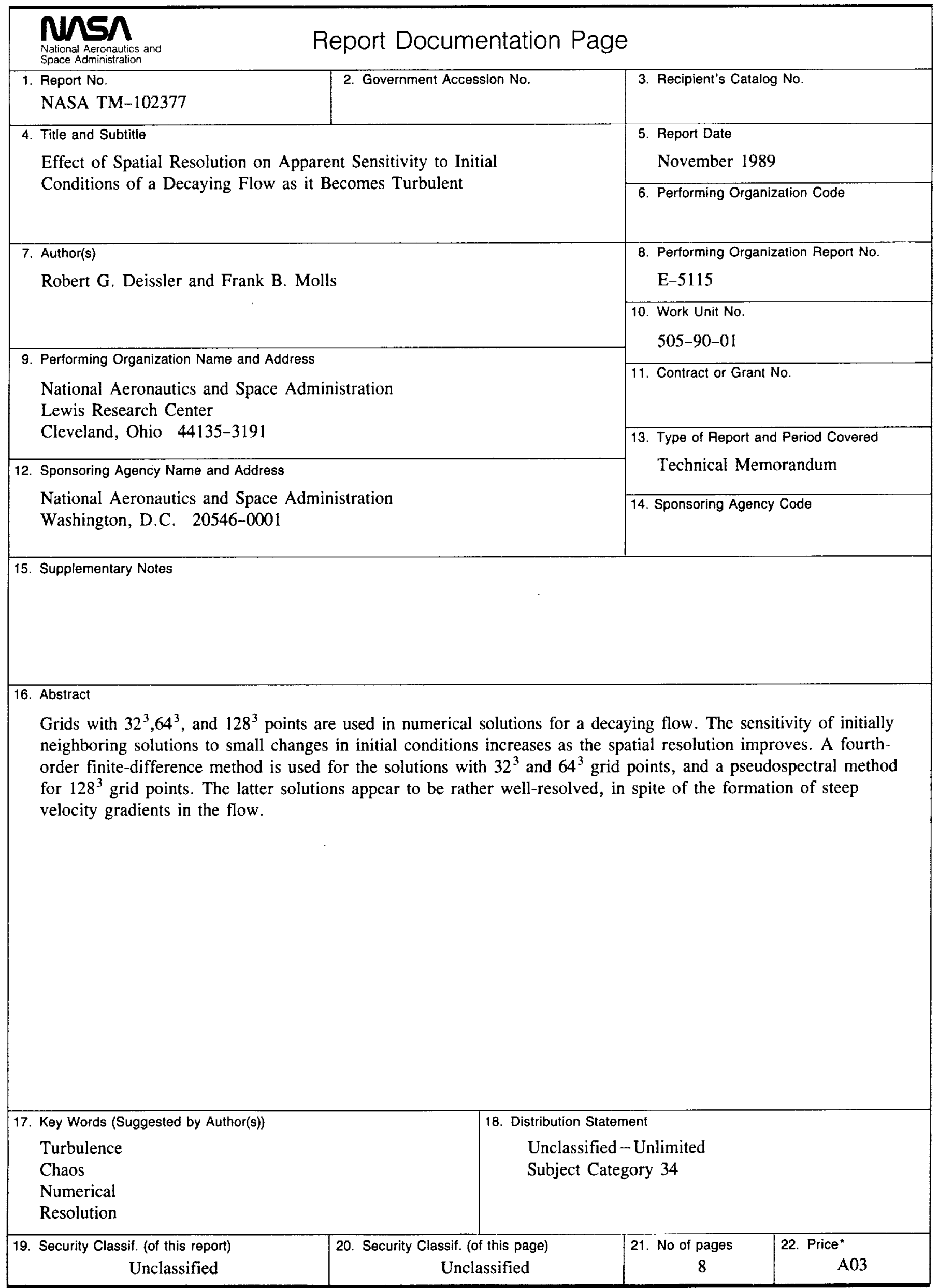


